\title{
Zinc Vacancy - Hydrogen Complexes as Major Defects in ZnO Nanowires Grown by Chemical Bath Deposition
}

José Villafuerte, ${ }^{1,2}$ Fabrice Donatini, ${ }^{2}$ Joseph Kioseoglou, ${ }^{3}$ Eirini Sarigiannidou, ${ }^{1}$ Odette ChaixPluchery, ${ }^{1}$ Julien Pernot, ${ }^{2}$ and Vincent Consonni ${ }^{*}$

${ }^{1}$ Univ. Grenoble Alpes, CNRS, Grenoble INP, LMGP, F-38000 Grenoble, France

${ }^{2}$ Univ. Grenoble Alpes, CNRS, Grenoble INP, Institut NEEL, F-38000 Grenoble, France

${ }^{3}$ Physics Department, Aristotle University of Thessaloniki, 54124 Thessaloniki, Greece

*Corresponding Author: vincent.consonni@grenoble-inp.fr

\section{SUPPLEMENTARY INFORMATION}

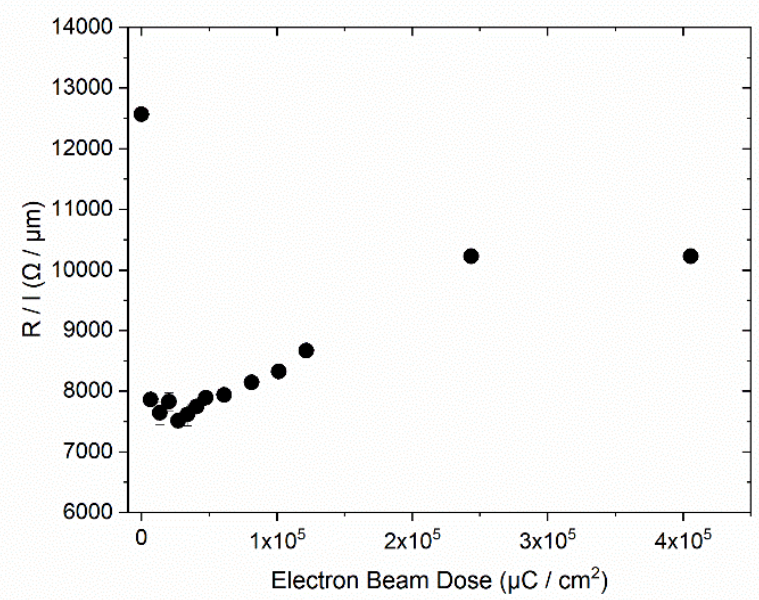

Figure S1. Typical evolution of $R / l$ as a function of the electron beam dose. 

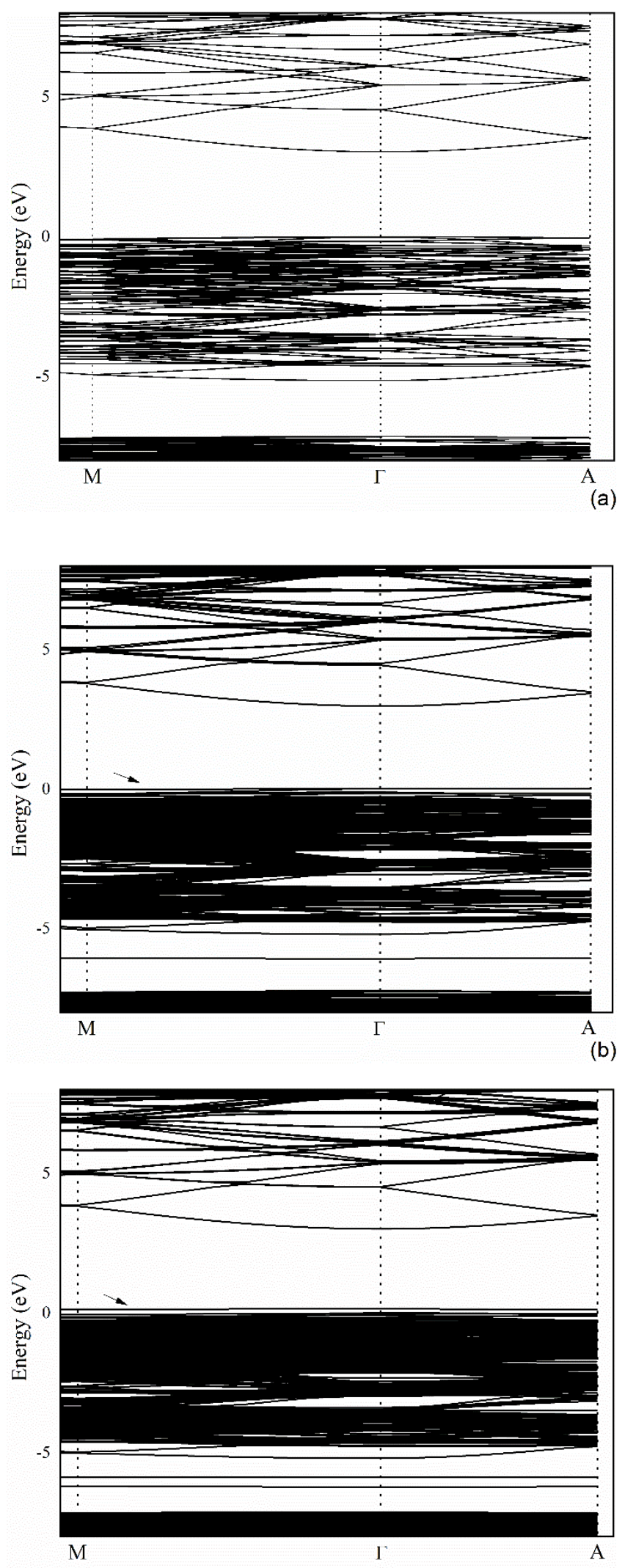

(c)

Figure S2. Electronic band structures of (a) the pristine $\mathrm{ZnO}$ supercell of 192 atoms, (b) the energetically favourable relaxed configuration of the supercell with one $\mathrm{VZn}$ and one $\mathrm{H}$ atom in 
the neutral charge state, and (c) the energetically favourable relaxed configuration of the supercell with one $V \mathrm{Zn}$ and two $\mathrm{H}$ atoms in the neutral charge state. The zero energy corresponds to the VBM in the prefect crystal. 